University of Wollongong

Research Online

Faculty of Social Sciences - Papers (Archive) Faculty of Arts, Social Sciences \& Humanities

$1-1-2018$

The relationship between executive functions and emotion regulation in females attending therapeutic community treatment for substance use disorder

Ely Marceau

University of Wollongong, emm993@uowmail.edu.au

Peter James Kelly

University of Wollongong, pkelly@uow.edu.au

Nadia Solowij

University of Wollongong, nadia@uow.edu.au

Follow this and additional works at: https://ro.uow.edu.au/sspapers

Part of the Education Commons, and the Social and Behavioral Sciences Commons

Research Online is the open access institutional repository for the University of Wollongong. For further information contact the UOW Library: research-pubs@uow.edu.au 


\title{
The relationship between executive functions and emotion regulation in females attending therapeutic community treatment for substance use disorder
}

\author{
Abstract \\ (C) 2017 Elsevier B.V. Background Difficulties in emotion regulation influence the development of \\ substance use disorder (SUD), its severity, course, treatment outcomes, and relapse. Impaired executive \\ functions (EFs) are common in SUD populations and may relate to emotion dysregulation. The current \\ study tested whether performance on three basic EF tasks ('working memory', 'inhibition', and 'task- \\ switching') and/or inventory-based assessment of EF were related to difficulties in emotion regulation in \\ females attending residential SUD therapeutic community treatment. Methods Cross-sectional design in \\ which participants ( $N=50$, all female) completed a questionnaire battery including the Difficulties in \\ Emotion Regulation Scale (DERS) and Behavior Rating Inventory of Executive Function - Adult Version \\ (BRIEF-A) was used. Participants also completed neuropsychological assessment of EF including the \\ Working Memory Index (WMl; Wechsler Adult Intelligence Scale), and measures of inhibition and task- \\ switching (Color-Word Interference Test; Delis-Kaplan Executive Function System). Results Executive \\ dysfunction, as assessed by the Global Executive Composite (GEC; BRIEF-A), and personality disorder \\ indicators (Standardised Assessment of Personality - Abbreviated Scale; SAPAS) were positively \\ correlated with DERS scores. Sequential hierarchical regression indicated that task-switching, GEC, and \\ SAPAS scores statistically predicted DERS scores, while working memory and inhibition did not. \\ Mediation analysis indicated that there was a significant indirect effect of GEC scores and task-switching \\ performance on DERS scores, through SAPAS scores. Conclusions Impairment of EF, particularly task- \\ switching, is related to difficulties in emotion regulation in a female sample attending residential SUD \\ treatment. Cognitive training interventions that improve task-switching performance may be beneficial in \\ promoting effective emotion regulation and improved SUD treatment outcomes.

\section{Disciplines} \\ Education | Social and Behavioral Sciences

\section{Publication Details} \\ Marceau, E., Kelly, P. \& Solowij, N. (2018). The relationship between executive functions and emotion \\ regulation in females attending therapeutic community treatment for substance use disorder. Drug and \\ Alcohol Dependence, 182 58-66.
}


The relationship between executive functions and emotion regulation in females attending therapeutic community treatment for substance use disorder

Ely M. Marceau ${ }^{\mathrm{a}}$, Peter J. Kelly ${ }^{\mathrm{a}}$, and Nadia Solowij ${ }^{\mathrm{a}}{ }^{*}$

${ }^{\text {a }}$ School of Psychology and Illawarra Health and Medical Research Institute,

University of Wollongong, Northfields Ave Wollongong NSW 2522 Australia

* Corresponding Author at:

School of Psychology, University of Wollongong

Northfields Ave Wollongong NSW 2522 Australia

Tel: +61242213732, Fax: +61242214163, E-mail: nadia@uow.edu.au 


\begin{abstract}
Background

Difficulties in emotion regulation influence the development of substance use disorder (SUD), its severity and course, treatment outcomes, and relapse. Impaired executive functions (EFs) are common in SUD populations and may relate to emotion dysregulation. The current study tested whether performance on three basic EF tasks ('working memory', 'inhibition', and 'task-switching') and/or inventory-based assessment of EF were related to difficulties in emotion regulation in females attending residential SUD therapeutic community treatment.

Methods
\end{abstract}

Cross-sectional design with participants $(N=50$, all female) completing a questionnaire battery including the Difficulties in Emotion Regulation Scale (DERS) and Behavior Rating Inventory of Executive Function - Adult Version (BRIEF-A). Participants also completed neuropsychological assessment of EF including the Working Memory Index (WMI; Wechsler Adult Intelligence Scale), and measures of inhibition and task-switching (Color-Word Interference Test; Delis-Kaplan Executive Function System).

Results

Executive dysfunction, as assessed by the Global Executive Composite (GEC; BRIEF-A), and personality disorder indicators (Standardised Assessment of Personality - Abbreviated Scale; SAPAS) were positively correlated with DERS scores. Sequential hierarchical regression indicated that task-switching, GEC, and SAPAS scores statistically predicted DERS scores, while working memory and inhibition did not. Mediation analysis indicated that there was a significant indirect effect of GEC scores and task-switching performance on DERS scores, through SAPAS scores.

\title{
Conclusions
}

Impairment of EF, particularly task-switching, is related to difficulties in emotion regulation in a female sample attending residential SUD treatment. Cognitive training interventions that improve task-switching performance may be beneficial in promoting effective emotion regulation and improved SUD treatment outcomes.

Keywords: Emotion Regulation; Executive Functions; Task-Switching; Substance Use Disorder; Therapeutic Community 


\section{Introduction}

When an emotion arises, a complex interplay between subjective experience, cognition, physiological changes, and behavioral components takes place, with neural bases such as the lateral prefrontal and parietal cortices and the ventral anterior cingulate and ventromedial prefrontal cortices implicated (Etkin et al., 2015). The pursuit of desired emotional states in everyday life is ubiquitous and "emotion regulation" denotes this process; that of modifying the intensity or duration of existing emotions in order to maintain goal-directed behavior (Tamir, 2016).

Emotion dysregulation is implicated in various forms of psychopathology (Aldao et al., 2010), including substance use disorder (SUD; Cheetham et al., 2010), in which it has a significant effect on the initiation, severity, and prognosis of the disorder (Wilcox et al., 2016). For example, the capacity for effective emotion regulation is under development during adolescence and may, in combination with other risk factors, increase the likelihood of early initiation of substance use, disruption of neuromaturational processes, and subsequent poor prognosis (Gladwin et al., 2011; Lubman et al., 2007; Smith and Cyders, 2016; Wills et al., 2016).

Individuals with affective disorders (e.g., anxiety and mood disorders) commonly experience comorbid SUD and, conversely, SUDs also contribute to the development of affective disorders (Cheetham et al., 2010). Additionally, SUD populations experience greater difficulties with emotion regulation relative to controls (Wilcox and Adinoff, 2015) and substance use may develop as a mechanism to relieve negative affect (Blevins et al., 2016). Emotion dysregulation increases the severity of SUD (Tull et al., 2015) and predicts relapse to substance use (Witkiewitz and $\mathrm{Wu}, 2010)$, while reductions in negative affect predict reduced SUD severity following treatment completion (Mo and Deane, 2016). 
Akin to the role of emotion dysregulation in SUD, cognitive deficits are commonly observed and also impede the treatment process. Cognitive impairment is one of the four most common risk factors for dropout from SUD treatment (Brorson et al., 2013) and the specific impairment of executive function (EF) is well documented in SUD populations (Fernández-Serrano et al., 2010; Hester et al., 2010).

Along with the challenges to effective SUD treatment associated with emotion dysregulation and cognitive deficits, comorbid personality disorder is a significant risk factor for early dropout (Brorson et al., 2013) and is highly prevalent in SUD populations (Grant et al., 2015; Grant et al., 2016). Further to this, personality disorder is independently associated with cognitive deficits, with the most commonly observed deficits in memory, decision-making, and EFs (Unoka \& Richman, 2016). While several conceptualisations of EF exist, Miyake et al. (2000) proposed the unity/diversity framework of EF, which posits three separable, basic EFs: 'working memory', 'inhibition', and 'task-switching' (Friedman and Miyake, 2017; Miyake and Friedman, 2012). Working memory refers to the capacity to monitor and alter the contents of working memory, inhibition is the ability to override an unwanted distraction to maintain task-focus, and task-switching involves flexibly switching attention between tasks or mental sets (Hofmann et al., 2012).

It has been proposed that these basic EFs (i.e., working memory, inhibition, and task-switching) may subserve effective emotion regulation (Hofmann et al., 2012; Schmeichel and Tang, 2015). The contributions of working memory, inhibition, and task-switching to emotion regulation have been explored in a number of studies, predominantly drawing from nonclinical populations. For example, a series of studies explored the role of working memory (assessed using the operation span task) in emotion regulation within a university student sample (Schmeichel and Demaree, 
2010; Schmeichel et al., 2008). Participants with a higher working memory capacity were better able to appraise emotional stimuli and, consequently, more effectively experience and express emotion (Schmeichel et al., 2008); these abilities were not confounded with higher working memory capacity leading to participants being better able to follow instructions (Schmeichel and Demaree, 2010). Another study in a community sample found that effective reappraisal of emotions was predicted by higher working memory capacity in a modified operation span task (McRae et al., 2012). The specific role of updating the contents of working memory in emotion regulation was explored in university students using the emotional 2-back task (Pe et al., 2015). Participants with better updating abilities displayed higher levels of emotional reactivity, but were more quickly and effectively able to regulate their emotions and return to a baseline level of arousal.

Several studies have demonstrated links between inhibition and emotion regulation. Inhibition performance, as measured by the Stroop task, was found to predict effective restraint of socially inappropriate behaviors in university students (von Hippel and Gonsalkorale, 2005). Similarly, university students who demonstrated poorer inhibition performance in the stop-signal task experienced larger increases in negative emotions following an emotion induction paradigm, relative to those with better inhibition performance (Tang and Schmeichel, 2014). In a sample of 5- to 7-year-old children, inhibition performance assessed by a go/no-go task predicted effort exerted in regulating emotions (Hudson and Jacques, 2014), with similar findings demonstrated in preschool-aged children (Carlson and Wang, 2007). In a clinical sample of patients with frontal lobe damage, inhibition performance via a go/no-go task mediated the relationship between prefrontal lobe damage and maladaptive emotion regulation strategies (Falquez et al., 2015). 
There is a paucity of studies investigating the role of task-switching in emotion regulation (Schmeichel and Tang, 2015). The previously mentioned study of working memory by McRae et al. (2012), also included a measure of task-switching (i.e., set-shifting costs based on a standardized global/local task), and found that as well as working memory capacity, task-switching also predicted effective reappraisal of emotions.

While a range of studies have investigated the role of working memory, inhibition, and, to a lesser extent, task-switching, these studies have primarily involved nonclinical populations and to the best of our knowledge, the role of these basic EFs in emotion regulation have not been explored in an SUD population. There is also a paucity of studies directly examining a relationship between EFs and emotion regulation in other psychiatric populations, despite clear deficits in each of these domains being widely reported (e.g., EF deficits in depression (Snyder, 2013); anxiety (de Lima Muller et al., 2015); bipolar disorder (Cullen et al., 2016); schizophrenia (Kluwe-Schiavon et al., 2013); post-traumatic stress disorder (Polak et al., 2012); borderline personality disorder (McClure et al., 2016); with emotion regulation deficits in these same populations (e.g., Gratz et al., 2016; Green et al., 2007; Joormann \& Stanton, 2016; Seligowski et al., 2015; van Zutphen et al., 2015; Zilverstand et al., 2016)).

While these basic performance-based EF tasks are sensitive to impairment of the frontal lobes (Jurado and Rosselli, 2007), some tasks may arguably have limited ecological validity and may not capture impaired functioning as effectively as inventory-based (i.e., self-report) assessment of EFs (Isquith et al., 2013). Furthermore, performance- and inventory-based measures of EFs are minimally correlated and may assess distinct components of EFs (Toplak et al., 2013). For 
example, inventory-based assessment of EFs, using the Behavior Rating Inventory of Executive Function - Adult Version (BRIEF-A; Roth et al., 2005), more effectively distinguished polysubstance users from controls and was more strongly associated with social adjustment outcomes, compared to performance-based measures (Hagen et al., 2016). In light of these considerations, the current study included both performance- and inventory-based assessment of EFs.

Given the importance of emotion regulation in SUD treatment and the potential connection between EFs and effective emotion regulation, the current study sought to ascertain whether inventory-based assessment of EFs, and/or performancebased assessment of working memory, inhibition, and task-switching performance were related to difficulties in emotion regulation in an all-female residential SUD population. A further aim of the current study was to explore the role of personality disorder in the relationship between EFs and emotion dysregulation, given its high prevalence in SUD populations, deleterious effect on SUD treatment, and independent association with cognitive deficits.

\section{Material and methods}

\subsection{Participants}

Participants $(N=50)$ were recruited from We Help Ourselves (WHOs), a large provider of residential SUD treatment in Australia, which utilises the Therapeutic Community model of treatment (De Leon, 1989). Inclusion criteria for the study

were: (i) diagnosis of substance abuse/dependence, based on DSM-IV-TR criteria ${ }^{1}$,

\footnotetext{
${ }^{1}$ DSM-5 criteria for substance use disorder do not separate abuse and dependence, but provide criteria for these specifiers. Despite this, DSM-5 criteria are almost identical to those used in DSM-IV-TR (with the exception of removal of recurrent legal problems associated with substance use criterion and the addition of craving or
} 
assessed using the Mini-International Neuropsychiatric Interview (MINI-Plus; Sheehan et al., 1998), (ii) a minimum abstinence period of 7 days (with confirmation of detoxification a prerequisite of entry to treatment), (iii) absence of any neurological, infectious, or other disease affecting the central nervous system (e.g., epileptic seizures, stroke, brain tumour, meningitis, encephalitis, multiple sclerosis, HIV positive), and (iv) English as native language. Limited leave from the residential facility, close observation by staff and other residents, and random urine tests assured abstinence (excluding caffeine and nicotine) during the course of participation in this study.

\subsection{Procedure}

Data from this study were drawn from baseline assessments of a subsequent study that investigated the effects of cognitive remediation in residents of an SUD therapeutic community (Marceau et al., 2017). All residents in the treatment facility were invited to participate in the study and the recruitment rate was $96 \%$. Participants were female, aged between 19 and 56 years $(M=32.5, S D=8.1)$, and had completed an average of 10.8 years of education $(S D=2.5$, range 6-20). Participants had been in treatment for an average of 46.9 days $(S D=38.9$, range 2-160).

\subsection{Screening, diagnostic and clinical assessment}

Diagnostic and clinical assessment at baseline included the following: Diagnoses of substance abuse/dependence and other current and past Axis I diagnoses were assessed using the MINI-Plus. Screening for personality disorder diagnoses was conducted using the Standardised Assessment of Personality - Abbreviated Scale (SAPAS; Moran et al., 2003), as shown to be an appropriate time-efficient and costeffective assessment for SUD populations (Gonzalez, 2014; Hesse and Moran, 2010;

strong desire to use a substance criterion, as well as slight changes in number of criteria required to meet diagnostic thresholds). 
Hesse et al., 2008). The SAPAS is an 8-item scale including questions such as, "In general, do you have difficulty making and keeping friends?" and "In general, do you trust other people?". Participants were instructed that the questions were about "how you behave and the way you think and feel things usually - in other words they are about your behavior and your way of being in general" and were asked to answer with a yes/no response. In the case of answering "yes" to a question, participants were then asked if their response applied "most of the time and in most situations" to which they also responded yes or no. SAPAS responses were scored as per the standardized instructions (i.e., one point assigned when participants endorsed both initial and follow-up questions). Scores $\geq 4$ are considered to be an indicator of personality disorder, as this threshold demonstrated correct classification of $73.6 \%$ of an inpatient SUD sample, with a sensitivity of $81.8 \%$ and a specificity of $67.7 \%$ (Gonzalez, 2014). The SAPAS demonstrated concurrent validity in an SUD sample, as it correlated with other personality disorder measures after controlling for psychiatric symptoms and recent substance use (Hesse and Moran, 2010). Additionally, the SAPAS demonstrated modest internal consistency and test-retest correlation, and was shown to correlate with clinician-report of externalising and global assessment of functioning (Hesse et al., 2008). The current study utilised SAPAS scores as a dimensional indicator of personality disorder, in light of research suggesting that personality disorder may be more accurately characterised as a dimensional, rather than categorical, construct (Karukivi et al., 2017). Higher scores indicated greater likelihood and severity of personality disorder.

\subsection{Measures}

2.4.1 Difficulties in Emotion Regulation Scale (DERS; Gratz and Roemer, 2004). The DERS is a 36-item self-report questionnaire that assesses clinically relevant 
difficulties in emotion regulation. Example items include: "When I'm upset, I become angry with myself for feeling that way"; "I experience my emotions as overwhelming and out of control". There are six subscales contributing to a total score, which was used as the outcome variable: Nonacceptance of Emotional Responses, Difficulties Engaging in Goal-Directed Behavior, Impulse Control Difficulties, Lack of Emotional Awareness, Limited Access to Emotion Regulation Strategies, and Lack of Emotional Clarity. Higher scores denoted greater difficulties in emotion regulation. The DERS has demonstrated good internal consistency in a number of studies of SUD samples (e.g., Di Pierro et al., 2015; Hopwood et al., 2015; Tull et al., 2012) and was positively correlated with amygdala D2-type dopamine receptor availability in methamphetamine users and healthy controls (Okita et al., 2016).

\subsubsection{Behavior Rating Inventory of Executive Function - Adult Version (BRIEF-A;}

Roth et al., 2005). The BRIEF-A is a 75-item self-report questionnaire consisting of nine subscales (Inhibit; Shift; Emotional Control; Self-Monitor; Initiate; Working Memory; Plan/Organize; Task Monitor; Organisation of Materials). Participants are instructed to answer each question by selecting "never", "sometimes", or "often", in relation to whether they have had problems with any of the listed behaviors in the past month. Example items include: "I don't plan ahead for tasks"; "I forget what I am doing in the middle of things". The outcome variable was the Global Executive Composite (GEC), which provides an overall summary score, with elevated scores indicating executive dysfunction. The BRIEF-A has been demonstrated as a sensitive measure of EFs in individuals with SUD (Hagen et al., 2016).

\subsubsection{Working memory: Working Memory Index (WMI; Wechsler Adult Intelligence} Scale, fourth edition: WAIS-IV; Wechsler, 2008). The WMI of the WAIS-IV assesses components of working memory and is comprised of 2 subtests, which were 
administered as per the standardized instructions. The digit span subtest requires participants to recall various sequences of numbers (forward, backward, and in sequence) and the arithmetic subtest involves participants solving numerical problems within 30 seconds, after they have been read aloud by the examiner. The outcome variable was computed by following the standard scoring instructions and was the sum of the 2 subtests, which yielded a scaled index score, with higher scores corresponding to better performance.

\subsection{2. Inhibition and task-switching: Color-Word Interference Test (Delis-} Kaplan Executive Function System: D-KEFS; Delis et al., 2001). These subtests of the D-KEFS assess response inhibition and task-switching. Participants are instructed to read the items presented in each of four conditions as quickly and accurately as possible. Performance is measured in time (seconds). The first condition presents patches of colors and requires participants to name the colors. The second condition presents the words "red", "blue", and "green" and requires participants to read the words. The third condition presents words printed in incongruent colors and requires the participant to ignore the word and say the color. The fourth condition presents words printed in incongruent colors and requires the participant to switch between two rules: (a) ignore the word and say the color; and (b) ignore the color and say the word. Outcome variables were scaled scores of inhibition (condition 3 scaled score) and task-switching (condition 4 scaled score minus condition 3 scaled score). Higher scores reflect better performance.

\subsection{Statistical methods}

All statistical analyses were performed using IBM Statistical Package for the Social Sciences (SPSS version 21; IBM Corp, 2012). Three missing values were identified, one within each of the DERS, inhibition, and task-switching variables. 
The data analysis strategy involved three stages. Initially, intercorrelations amongst study variables were calculated, with a Bonferroni correction for multiple comparisons $(p<.05 / 45=p<.001)$. Spearman's rank-order correlations were performed as Shapiro-Wilk statistics indicated non-normal distributions for some variables. Subsequently, a sequential hierarchical regression was employed with DERS scores as the dependent variable, with the goal of ascertaining the role of unique EFs and other variables of interest in predicting emotion dysregulation. Sample size was chosen on the basis of utilising the largest available sample at baseline in a study that subsequently investigated the effects of a cognitive remediation intervention vs. treatment as usual (Marceau et al., 2017). In guiding number of variables included in the regression analyses, it has been suggested that including two subjects per variable is adequate (Austin \& Steyerberg, 2015). All statistical assumptions were tested and satisfied (i.e., independence of observations; linear relationships between variables; homoscedasticity of residuals; multicollinearity; outliers, high leverage points, highly influential points; normal distribution of residuals). In the final stage, four mediation models based on the outcomes of the regression analysis were tested in order to explore the influence of comorbid personality disorders on the relationship between EFs and emotion regulation. The mediation models were tested using the PROCESS macro (release 2.16.2; Hayes, 2013), recommended as an alternative to the causal step approach (Hayes, 2009; Hayes and Rockwood, 2016), and indirect effect was calculated using a bias-corrected and accelerated (BCa) bootstrapped confidence interval (CI) method, based on 5000 samples. Bonferroni correction was utilised to account for multiple comparisons $(p<.05 / 4=p<.0125)$. 


\section{Results}

Socio-demographic and substance use characteristics of the sample are displayed in Table 1, along with emotion regulation and EF measures. Participants were, on average, in their early thirties $(\mathrm{Mdn}=32.5$ years, range 19-56) and had completed approximately 11 years of education $(\mathrm{Mdn}=10.5$ years, range 6-20). The majority of participants (44\%) reported methamphetamine as their primary substance of misuse, followed by approximately $30 \%$ who reported alcohol, with smaller numbers of amphetamines, heroin, cannabis, and sedatives users. However, high rates of polysubstance use history were evident, with participants reporting using multiple substances over a number of years. With regard to EFs, single sample $t$-tests determined that relative to standardised normative scores, the sample scored significantly lower on the WMI, $t(49)=-6.42, p<.001$, and had significantly higher GEC scores, $t(49)=6.17, p<.001$, indicating higher levels of executive dysfunction. Table 2 displays psychiatric comorbidities, with the majority of the sample (64\%) meeting criteria for both current and past Axis I diagnoses, and $40 \%$ meeting the screening threshold for a personality disorder diagnosis. Significant heterogeneity of psychiatric comorbidities was observed. The sample was representative of individuals in therapeutic community treatment for SUD (Darke et al., 2012).

Table 3 displays Spearman's rank-order correlations between all continuous study variables. DERS scores were significantly positively correlated with SAPAS and GEC scores. No significant correlations were found between DERS scores and age, education, treatment length, WMI scores, or inhibition performance, although a nonsignificant trend was observed for a negative association with task-switching performance (rho=-0.37). Education was significantly correlated with WMI scores and was therefore included as a predictor variable. Similarly, although task-switching, 
WMI, and inhibition performance were not significantly correlated with DERS scores, they were also included as predictor variables in light of the study aim of ascertaining the unique role of EFs in predicting difficulties in emotion regulation, based on the theoretical framework of Hofmann et al. (2012).

\section{Table 1}

Socio-demographic, substance use, and psychiatric comorbidity characteristics, and emotion regulation and executive functions measures of residents of a female-only substance use therapeutic community

\begin{tabular}{|c|c|}
\hline Characteristic & $N=50$ \\
\hline Age $(M, S D)$ & $32.5(8.1)$ \\
\hline Education $(M, S D)$ & $10.8(2.5)$ \\
\hline Unemployed (\%) & 78.0 \\
\hline Marital status (\% single) & 74.0 \\
\hline Treatment length $(M, S D)$ & $46.9(38.9)$ \\
\hline \multicolumn{2}{|l|}{ Primary substance of misuse $(\%)$} \\
\hline Methamphetamine & 44.0 \\
\hline Alcohol & 28.0 \\
\hline Amphetamines & 8.0 \\
\hline Heroin & 8.0 \\
\hline Cannabis & 6.0 \\
\hline Sedatives & 6.0 \\
\hline \multicolumn{2}{|l|}{ Years of regular use $(M, M d n, S D ; n)$} \\
\hline Alcohol (any use) & $11.2(10.0,8.3 ; 43)$ \\
\hline Alcohol (to intoxication) & $9.6(6.5,7.6 ; 42)$ \\
\hline Heroin & $9.9(8.0,8.3 ; 9)$ \\
\hline Methadone & $7.3(7.0,5.7 ; 5)$ \\
\hline Other opiates/analgesics & $7.4(7.0,5.7 ; 11)$ \\
\hline Sedatives/hypnotics/tranquilisers & $8.7(10.0,6.3 ; 17)$ \\
\hline Cocaine & $6.6(6.0,5.6 ; 11)$ \\
\hline Amphetamines & $8.2(8.0,5.5 ; 42)$ \\
\hline Cannabis & $11.1(10.0,7.0 ; 43)$ \\
\hline Hallucinogens & $5.3(3.0,5.1 ; 9)$ \\
\hline More than one substance per day & $9.9(10.0,6.2 ; 41)$ \\
\hline SAPAS $(M, S D)$ & $3.0(1.9)$ \\
\hline $\operatorname{DERS}(M, S D)$ & $91.9(25.2)$ \\
\hline $\operatorname{GEC}(M, S D)$ & $61.8(13.5)$ \\
\hline
\end{tabular}


Task-switching $^{\mathrm{a}}(M, S D)$ $10.2(2.8)$

DERS Difficulties in Emotion Regulation Scale, SAPAS Standardised Assessment of Personality Abbreviated Scale, GEC Global Executive Composite: Behavior Rating Inventory of Executive Function - Adult version (BRIEF-A), WMI Working Memory Index: Wechsler Adult Intelligence Scale, fourth edition (WAIS-IV), Inhibition Condition 3 scaled score; Color-Word Interference Test: Delis-Kaplan Executive Function System (D-KEFS), Task-switching Condition 4 - Condition 3 contrast scaled score; Color-Word Interference Test: Delis-Kaplan Executive Function System (DKEFS) ${ }^{\text {a }} N=49$. 


\section{Table 2}

Psychiatric comorbidities of residents of a female-only substance use therapeutic community

$\begin{array}{ll}\text { Psychiatric Comorbidity }^{\mathrm{a}} & N=50\end{array}$

Current Axis I psychiatric diagnosis (\%)

64.0

Past Axis I psychiatric diagnosis (\%)

64.0

Personality disorder screen (met criteria) ${ }^{\mathrm{b}}(\%)$

40.0

Major depressive episode

Never (lifetime) $\quad 46.0$

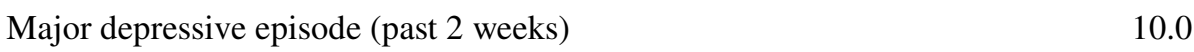

Substance-induced mood disorder (past 2 weeks) 2.0

Mood disorder due to medical condition (past 2 weeks) 2.0

$\begin{array}{ll}\text { Past major depressive episode } & 36.0\end{array}$

Past mood disorder due to medical condition $\quad 4.0$

Dysthymia

Never (lifetime)

Dysthymia (past 2 years)

Past dysthymia

Manic episode

Never (lifetime)

80.0

Current manic episode

0.0

Past manic episode

Past hypomanic episode

6.0

Past substance-induced hypomanic episode

Past hypomanic episode due to medical condition

Panic disorder

Never (lifetime)

60.0

Panic disorder (past month) $\quad 6.0$

Substance-induced anxiety disorder with panic attacks (past month) $\quad 4.0$

Anxiety disorder with panic attacks due to a medical condition (past month) $\quad 6.0$

$\begin{array}{lr}\text { Panic disorder (lifetime) } & 8.0\end{array}$

$\begin{array}{lr}\text { Panic disorder symptoms (lifetime) } & 16.0\end{array}$

Agoraphobia

Never (lifetime)

66.0

Current agoraphobia 20.0

$\begin{array}{ll}\text { Agoraphobia (lifetime) } & 14.0\end{array}$

$\begin{array}{ll}\text { Social phobia (past month) } & 20.0\end{array}$

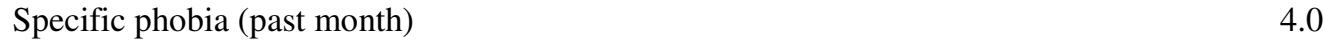

Obsessive compulsive disorder (past month) $\quad 2.0$

Posttraumatic stress disorder (past month) $\quad 14.0$

$\begin{array}{ll}\text { Substance-induced psychotic disorder (lifetime) } & 26.0\end{array}$ 
Anorexia nervosa (past 3 months)

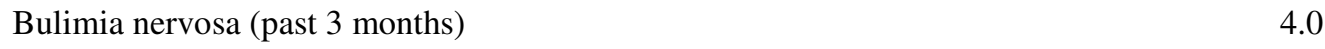

Generalized anxiety disorder (past 6 months) $\quad 18.0$

Substance-induced generalised anxiety disorder $\quad 8.0$

Generalised anxiety disorder due to medical condition $\quad 2.0$

Antisocial personality disorder (lifetime) $\quad 20.0$

$\begin{array}{ll}\text { Somatization disorder (lifetime) } & 0.0\end{array}$

$\begin{array}{ll}\text { Hypochondriasis (past } 6 \text { months) } & 2.0\end{array}$

$\begin{array}{ll}\text { Pain disorder (current) } & 0.0\end{array}$

Adult attention deficit/hyperactivity disorder (lifetime) $\quad 18.0$

Probable premenstrual dysphoric disorder (past year) $\quad 18.0$

${ }^{a}$ Mini-International Neuropsychiatric Interview. ${ }^{b}$ Standardised Assessment of Personality Abbreviated Scale. 
Predictor variables for the sequential hierarchical regression were entered in the following order: years of education (Step 1); current Axis I diagnosis (excluding SUD; Step 2); global self-report measure of EFs: GEC scores (Step 3); three specific EF components: performance in WMI, inhibition, and task-switching tasks (Step 4); and personality disorder indicators, SAPAS (Step 5). At step 1, the model was nonsignificant, $F(1,46)=0.16, p=.690$, with education accounting for only $0.03 \%$ of the variance in DERS, $R=.059, R^{2}=.003$. At step 2, presence of a current Axis I diagnosis (other than SUD) significantly improved prediction, explaining an additional $27.1 \%$ of the variance, $R=.524, R^{2}=.274, F$ change $(1,45)=16.78, p$ $<.001$. At step 3, the addition of GEC scores significantly improved prediction and explained an additional $11.7 \%$ of the variance, $R=.625, R^{2}=.291, F$ change $(1,44)=$ 8.44, $p=.006$. At step 4, the addition of WMI, inhibition, and task-switching performance significantly improved prediction and explained an additional $16.3 \%$ of the variance, $R=.745, R^{2}=.554, F$ change $(3,41)=5.01, p=.005$. At the final step, the addition of SAPAS scores significantly improved prediction and explained a further $4.4 \%$ of the variance, $R=.774, R^{2}=.599, F$ change $(1,40)=4.44, p=.042$. Table 4 displays unstandardized regression coefficients (B), standardized regression coefficients $(\beta), t$ scores, and $R^{2}$ change for all variables at each step, capturing the proportion of the variance in DERS scores uniquely explained by each variable at respective points of entry. In the final model task-switching performance, GEC scores, and SAPAS scores contributed to DERS scores in descending order of statistical significance. A post-hoc power analysis for the sequential hierarchical regression model was conducted using the software package, G*Power version 3.1 (Faul et al., 2009). The partial $R^{2}$ value of .53 and sample size of 49 were used in the analysis, with 7 predictor variables, and 5 tested predictor variables, and alpha set at $p<.05$. 
Results indicated that statistical power exceeded .99 for the detection of a large effect, suggesting adequate power.

In light of these regression results highlighting the contribution of EFs in general, and the task-switching component of EFs in particular in the prediction of DERS scores, a series of Bonferroni-corrected mediation analyses were employed to examine the relationship between EFs and difficulties in emotion regulation. Specifically, the role

\section{Table 4}

Regression coefficients of education, executive functions, and psychiatric comorbidities in predicting Difficulties in Emotion Regulation Scale (DERS) scores

\begin{tabular}{|c|c|c|c|c|c|}
\hline & Variable & $B$ & $\beta$ & $t$ & $R_{\text {change }}^{2}$ \\
\hline Step 1 & Education & 0.60 & .06 & 0.40 & .003 \\
\hline \multirow[t]{2}{*}{ Step 2} & Education & -1.01 & -.10 & -0.74 & .271 \\
\hline & Axis I diagnosis & 28.26 & .54 & $4.10 * * *$ & \\
\hline \multirow[t]{3}{*}{ Step 3} & Education & -1.23 & -.12 & -0.98 & .117 \\
\hline & Axis I diagnosis & 15.51 & .30 & 2.00 & \\
\hline & GEC & 0.79 & .43 & $2.90 * *$ & \\
\hline \multirow[t]{6}{*}{ Step 4} & Education & -2.50 & -.25 & -1.83 & .163 \\
\hline & Axis I diagnosis & 13.39 & -.26 & 1.91 & \\
\hline & GEC & 0.73 & .39 & $2.96 * *$ & \\
\hline & WMI & 0.54 & .27 & 1.84 & \\
\hline & Inhibition & -2.20 & -.29 & $-2.03 *$ & \\
\hline & Task-switching & -3.99 & -.45 & $-3.48 * * *$ & \\
\hline \multirow[t]{7}{*}{ Step 5} & Education & -2.28 & -.22 & -1.74 & .044 \\
\hline & Axis I diagnosis & 10.01 & .19 & 1.45 & \\
\hline & GEC & 0.67 & .36 & $2.83 * *$ & \\
\hline & WMI & 0.34 & .17 & 1.16 & \\
\hline & Inhibition & -2.02 & -.26 & -1.92 & \\
\hline & Task-switching & -3.53 & -.40 & $-3.14 * *$ & \\
\hline & SAPAS & 3.46 & .26 & $2.11 *$ & \\
\hline
\end{tabular}

$\overline{B \text { unstandardized regression coefficients, } \beta \text { standardized regression coefficients, } G E C \text { Global Executive }}$ Composite: Behavior Rating Inventory of Executive Function - Adult version (BRIEF-A), WMI

Working Memory Index: Wechsler Adult Intelligence Scale, fourth edition (WAIS-IV), Inhibition

Condition 3 scaled score; Color-Word Interference Test: Delis-Kaplan Executive Function System (D- 
KEFS), Task-switching Condition 4 - Condition 3 contrast scaled score; Color-Word Interference Test: Delis-Kaplan Executive Function System (D-KEFS), SAPAS Standardised Assessment of Personality Abbreviated Scale

$* p \leq .05 ; * * p \leq .01 ; * * * p \leq .001$

of personality disorder, as indexed by SAPAS scores, was considered as a mediator. As shown in Figure 1, there was a significant indirect effect of GEC scores and taskswitching performance on DERS scores, through SAPAS scores. The ratio of the indirect effect to the total effect was calculated to provide a measure of effect size: $P_{M}$ $=a b \div c\left(\right.$ Wen and Fan, 2015); GEC $P_{M}=0.22$; task-switching $P_{M}=0.27$. Further mediation models indicated that neither WMI nor inhibition performance predicted DERS scores (see Figures 2 and 3), although WMI performance predicted SAPAS scores $(b=0.06, p=.002)$, and there was a trend toward inhibition performance predicting SAPAS scores $(b=0.15, p=.077)$. Additionally, SAPAS scores predicted DERS scores in both WMI $(b=7.06, p<.001)$ and inhibition $(b=6.92, p<.001)$ mediation models.

\section{Discussion}

This study sought to determine the relationships between working memory, inhibition, and task-switching performance, as well as inventory-based assessment of EFs and difficulties in emotion regulation in an all-female residential SUD sample attending therapeutic community treatment. This aim was based upon the theoretical framework proposing that the capacity for effective emotion regulation may be subserved by performance in basic EF tasks (Hofmann et al., 2012; Schmeichel and Tang, 2015). Results indicated that inventory-based executive dysfunction and personality disorder indicator scores were positively correlated with difficulties in 
emotion regulation. Additionally, task-switching performance was negatively correlated with emotion regulation difficulties. In the final hierarchical regression model, task-switching, executive dysfunction, and personality disorder indicator scores had the strongest associations with difficulties in emotion regulation.

Subsequent to this, four mediation models were employed to examine the relationship between inventory- and performed-based EFs, and difficulties in emotion regulation. There was a significant indirect effect of inventory-based EFs and task-switching performance, respectively, on difficulties in emotion regulation, through personality disorder indicator scores. This suggests that the relationship between both inventorybased assessment of executive dysfunction and deficits in task-switching performance, respectively, and difficulties in emotion regulation may be partly explained by the role of personality disorder.

Emotion regulation plays a crucial role in the development, severity, treatment outcomes, and prognosis of SUD (Wilcox et al., 2016), and increased understanding of corresponding neurocognitive impairments and the discovery of neurobiological substrates may serve to advance assessment strategies and the development of interventions that target emotion dysregulation in SUD treatment. Findings from the current study indicate that, compared to other basic performance-based EFs (i.e., working memory and inhibition), task-switching was uniquely associated with difficulties in emotion regulation. Additionally, this relationship was partly explained by personality disorder. Personality disorder was found to explain additional variance beyond the effect of control variables and EFs measures in its relationship to difficulties in emotion regulation. A potential explanation for this is that while evidence indicates significant EF deficits in personality disorder populations (GarciaVillamisar et al., 2017), impairments in decision-making, memory, visuospatial, 
processing speed, and verbal intelligence domains are also commonly observed (Unoka \& Richman, 2016), and may be related to emotion dysregulation. An alternative explanation is that other factors related to personality disorder (e.g., specific symptoms, interpersonal style) further contribute to difficulties in emotion regulation independently of cognitive deficits. Given that personality disorder has been identified as one of the top four risk factors for dropout from SUD treatment (Brorson et al., 2013), there is a pressing need for adjunct interventions and modifications to treatment that bolster individuals with co-occurring SUD, personality disorder, and/or cognitive deficits, and increase the likelihood of treatment engagement and completion.

Along with the unique role of task-switching as a performance-based EF that was associated with difficulties in emotion regulation, inventory-based assessment of executive dysfunction was also uniquely related to difficulties in emotion regulation, with this relationship partly explained by personality disorder. This finding is congruent with research suggesting that inventory- and performance-based measures of EFs may assess distinct components of EFs (Hagen et al., 2016; Toplak et al., 2013).

Recently, a number of studies have explored the potential for cognitive screening (e.g., Marceau et al., 2016) and training/remediation interventions to respectively facilitate the detection of cognitive dysfunction, and improve cognition, functional outcomes, and treatment retention in SUD populations (Manning et al., 2017; Marceau et al., 2017; Verdejo-García, 2016). Four main classes of interventions have been trialled based on two major proposed neuroscientific mechanisms: (i) reorienting biases towards impulsive action (i.e., bottom-up approach) and (ii) strengthening EFs (i.e., top-down approach), with cognitive bias modification and 
response inhibition training located within the former category, and working memory training and goal-directed interventions in the latter (Verdejo-García, 2016). Identifying the neurobiological mechanisms of these interventions is an area currently under investigation, but it is proposed that all interventions share the common underlying elements of normalising dysfunctional activity related to reward circuitry and also the strengthening of the inhibitory control network (Zilverstand et al., 2016). A range of cognitive domains have been targeted through cognitive training interventions for SUD, including working memory, inhibition, attention, sustained attention, logical reasoning, decision-making, and broad EFs (Marceau et al., 2017; Verdejo-García, 2016). Gaining a more in-depth understanding of the specific active ingredients of these interventions and how they relate to improvements in neurocognitive performance as well as meaningful clinical outcomes is an important area for further exploration. Given that findings from the current study suggest a relationship between task-switching and emotion regulation, future studies may wish to investigate this further, by assessing whether cognitive training interventions that specifically target improvement in task-switching performance are efficacious within SUD treatment and whether they also lead to improved emotion regulation capacity.

As per the recommendations of Hofmann et al. (2012), future research may seek to further examine the relationships between basic EFs and measures of selfregulation more broadly, using a number of proposed theoretical models. Specific to the addiction field, this line of research may provide significant evidence that could serve to inform the development of targeted cognitive training interventions. For example, future studies could examine the relationships between basic EFs (i.e., working memory, inhibition, task-switching) and a range of other self-regulation outcomes (e.g., self-control, responding to cravings in the context of substance-cues, 
etc.), to explore potential nuances involving mediating or moderating factors. This endeavour may be enhanced by employing measures that capture data from multiple levels of analysis (e.g., self-report, neurocognitive, neuroimaging; for a recent example investigating self-control in a nonclinical population, refer to Paschke et al., 2016). Furthermore, the inclusion of functional outcomes pertaining to SUD treatment and recovery in studies exploring the relationships between basic EFs and selfregulation may enhance this line of research and lead to fruitful, clinically relevant findings.

Performance on EFs tasks is partly shaped by individual differences (Miyake and Friedman, 2012). Additionally, individual factors also shape the trajectory of cognitive recovery in SUD (Bates et al., 2013). There are a variety of pathways leading to the onset of drug and alcohol addiction based on pre-existing individual differences in brain functioning; namely susceptibility to increased or decreased sensitivity in a range of executive systems in the brain (George and Koob, 2010). In relation to this, future studies may wish to examine the relationship between EFs, selfregulation, and the transition to addiction, while ensuring sufficient power to explore mediating and moderating factors based on the characteristics of particular subgroups that reflect these individual differences in the often-heterogeneous SUD populations.

The limitations of the current study must be noted. First, the small sample utilised exclusively consisted of females in residential SUD treatment. Future research may wish to explore the relationship between basic EFs and emotion regulation in male or mixed samples to ascertain whether similar or contrasting relationships exist. This is particularly important given that substance-related changes in cognition are influenced by sex (Becker et al., 2017), and gender differences potentially lead to diversity in SUD treatment response (Becker et al., 2016). Additionally, the current 
study utilised a screening measure of personality disorder and, although evidence suggests that this is an appropriate brief and cost-effective measure for SUD populations (Gonzalez, 2014; Hesse and Moran, 2010; Hesse et al., 2008), future studies may wish to examine the relationship between basic EFs and emotion regulation using full diagnostic assessments and/or other dimensional measures (Karukivi et al., 2017) of personality disorder. With regards to Axis I diagnoses, a high prevalence and significant heterogeneity was observed in the current study. While the current study aimed to explore the role of personality disorders, future studies with larger samples may explore the role of Axis I diagnoses in the relationship between EFs and emotion dysregulation (e.g., utilising mediation/moderation analyses to explore relationships for particular diagnostic subgroups).

A further area of exploration for future studies concerns assessment of cognition more broadly, through utilising neuropsychological batteries including diverse tasks. This could provide data to effectively characterise level of cognitive functioning, as well as performance across other cognitive domains. While SUD populations generally display cognitive deficits (e.g., Manning et al., 2017), it may be that the relationship between EFs and emotion dysregulation is influenced by overall level of cognitive performance and/or performance in other cognitive domains.

While there is some evidence suggesting that the self-report Difficulties in Emotion Regulation Scale (DERS) is appropriate for use in SUD populations, it must be acknowledged that due to the lack of large-scale validation studies in SUD populations, the validity and reliability of this measure for SUD remains to be clarified. A recent validation study for the DERS investigated a naturalistic sample of adult outpatients receiving dialectical behavior therapy including those with 
borderline personality disorder, mood disorder, anxiety disorder, and SUD diagnoses (Osborne et al., 2017). Results indicated that a modified bifactor model, which excluded items from the Lack of Emotional Awareness subscale, provided the best fit of the data. Additionally, DERS total score (excluding Lack of Emotional Awareness items) displayed acceptable reliability and criterion-related validity in the study sample. These findings highlight the need to clarify whether SUD populations display levels of insight and awareness regarding their emotional experience to render the use of a self-report measure of emotion dysregulation appropriate.

Related to this, there is a more general ongoing debate regarding the use of self-report measures vs. behavioral laboratory tasks (Cyders \& Coskunpinar, 2011). For example, it has been suggested that these methodologies may assess distinct components of the construct of impulsivity (Cyders \& Coskunpinar, 2011). Future research would benefit from the inclusion of a combination of experimental tasks assessing emotion regulation capacity, as well as self-report measures. This may help to clarify points of convergence and divergence of these methodologies, and provide a richer understanding of the relationship between EFs and emotion dysregulation in SUD populations.

In conclusion, the current study demonstrated that task-switching performance in contrast to other basic EFs, working memory and inhibition, was uniquely related to difficulties in emotion regulation in an all-female sample of residents attending residential SUD treatment. This relationship was partly explained by personality disorder indicators. These findings have implications for the design and development of cognitive training interventions to address the neurocognitive dysfunction present in SUD populations. There is a pressing need for further research that seeks to explore the neurocognitive bases of self-regulation to support the development of cognitive 
training interventions that include efficacious and specific active ingredients. The development of new interventions that translate to improvements in clinically meaningful outcomes that support treatment retention in SUD, such as emotion regulation, may benefit those individuals seeking long-term recovery from drug and alcohol addiction. 


\section{References}

Aldao, A., Nolen-Hoeksema, S., Schweizer, S., 2010. Emotion-regulation strategies across psychopathology: A meta-analytic review. Clin. Psychol. Rev. 30, 217-237.

Austin, P.C., Steyerberg, E.W., 2015. The number of subjects per variable required in linear regression analyses. J. Clin. Epidemiol. 68, 627-636.

Bates, M.E., Buckman, J.F., Voelbel, G.T., Eddie, D., Freeman, J., 2013. The mean and the individual: Integrating variable-centered and person-centered analyses of cognitive recovery in patients with substance use disorders. Front. Psychiatry 4, Article 177.

Becker, J.B., McClellan, M., Reed, B.G., 2016. Sociocultural context for sex differences in addiction. Addict. Biol. 21, 1052-1059.

Becker, J.B., McClellan, M.L., Reed, B.G., 2017. Sex differences, gender and addiction. J. Neurosci. Res. 95, 136-147.

Blevins, C.E., Abrantes, A.M., Stephens, R.S., 2016. Motivational pathways from antecedents of alcohol use to consequences: A structural model of using alcohol to cope with negative affect. Am. J. Drug Alcohol Abuse 42, 395-403.

Brorson, H.H., Ajo Arnevik, E., Rand-Hendriksen, K., Duckert, F., 2013. Drop-out from addiction treatment: A systematic review of risk factors. Clin. Psychol. Rev. 33, 1010-1024.

Carlson, S.M., Wang, T.S., 2007. Inhibitory control and emotion regulation in preschool children. Cogn. Dev. 22, 489-510.

Cheetham, A., Allen, N.B., Yücel, M., Lubman, D.I., 2010. The role of affective dysregulation in drug addiction. Clin. Psychol. Rev. 30, 621-634.

Cullen, B., Ward, J., Graham, N.A., Deary, I.J., Pell, J.P., Smith, D.J., Evans, J.J., 2016. Prevalence and correlates of cognitive impairment in euthymic adults with bipolar disorder: A systematic review. J. Affect. Disord. 205, 165-181.

Cyders, M.A., Coskunpinar, A., 2011. Measurement of constructs using self-report and behavioral lab tasks: Is there overlap in nomothetic span and construct representation for impulsivity? Clin. Psychol. Rev. 31, 965-982.

Darke, S., Campbell, G., Popple, G., 2012. Retention, early dropout and treatment completion among therapeutic community admissions. Drug Alcohol Rev. 31, 64-71.

De Leon, G., 1989. Therapeutic communities for substance abuse: Overview of approach and effectiveness. Psychol. Addict. Behav. 3, 140-147.

de Lima Muller, J., Torquato, K.I., Manfro, G.G., Trentini, C.M., 2015. Executive functions as a potential neurocognitive endophenotype in anxiety disorders: A systematic review considering DSM-IV and DSM-5 diagnostic criteria classification. Dement. Neuropsychol. 9, 285-294. 
Delis, D.C., Kaplan, E., Kramer, J.H., 2001. Delis-Kaplan Executive Function System: D-KEFS. The Psychological Corporation, San Antonio; Texas.

Di Pierro, R., Benzi, I.A., Madeddu, F., 2015. Difficulties in emotion regulation among inpatients with substance use disorders: The mediating effect of mature defense mechanisms. Clin. Neuropsychiatry 12, 83-89.

Etkin, A., Büchel, C., Gross, J.J., 2015. The neural bases of emotion regulation. Nature Rev. Neurosci. 16, 693-700.

Falquez, R., Dinu-Biringer, R., Stopsack, M., Arens, E.A., Wick, W., Barnow, S., 2015. Examining cognitive emotion regulation in frontal lobe patients: The mediating role of response inhibition. NeuroRehabilitation 37, 89-98.

Faul, F., Erdfelder, E., Buchner, A., Lang, A.-G., 2009. Statistical power analyses using $\mathrm{G}^{*}$ Power 3.1: Tests for correlation and regression analyses. Behav. Res. Methods 41, 1149-1160.

Fernández-Serrano, M.J., Pérez-García, M., Perales, J.C., Verdejo-García, A., 2010. Prevalence of executive dysfunction in cocaine, heroin and alcohol users enrolled in therapeutic communities. Eur. J. Pharmacol. 626, 104-112.

Friedman, N.P., Miyake, A., 2017. Unity and diversity of executive functions: Individual differences as a window on cognitive structure. Cortex 86, 186-204.

Garcia-Villamisar, D., Dattilo, J., Garcia-Martinez, M., 2017. Executive functioning in people with personality disorders. Curr. Opin. Psychiatry 30, 36-44.

George, O., Koob, G.F., 2010. Individual differences in prefrontal cortex function and the transition from drug use to drug dependence. Neurosci. Biobehav. Rev. 35, 232247.

Gladwin, T.E., Figner, B., Crone, E.A., Wiers, R.W., 2011. Addiction, adolescence, and the integration of control and motivation. Dev. Cogn. Neurosci. 1, 364-376.

Gonzalez, C., 2014. Screening for personality disorder in drug and alcohol dependence. Psychiat. Res. 217, 121-123.

Grant, B.F., Goldstein, R.B., Saha, T.D., Chou, S.P., Jung, J., Zhang, H., . . Hasin, D.S., 2015. Epidemiology of DSM-5 Alcohol Use Disorder: Results from the National Epidemiologic Survey on Alcohol and Related Conditions-III. JAMA Psychiatry 72, 757-766.

Grant, B.F., Saha, T.D., Ruan, W.J., Goldstein, R.B., Chou, S.P., Jung, J., . . Hasin, D.S., 2016. Epidemiology of DSM-5 Drug Use Disorder: Results from the National Epidemiologic Survey on Alcohol and Related Conditions-III. JAMA Psychiatry 73, $39-47$.

Gratz, K.L., Roemer, L., 2004. Multidimensional assessment of emotion regulation and dysregulation: Development, factor structure, and initial validation of the Difficulties in Emotion Regulation Scale. J. Psychopathol. Behav. Assess. 26, 41-54. 
Green, M.J., Cahill, C.M., Malhi, G.S., 2007. The cognitive and neurophysiological basis of emotion dysregulation in bipolar disorder. J. Affect. Disord. 103, 29-42.

Hagen, E., Erga, A.H., Hagen, K.P., Nesvåg, S.M., McKay, J.R., Lundervold, A.J., Walderhaug, E., 2016. Assessment of executive function in patients with substance use disorder: A comparison of inventory- and performance-based assessment. J. Subst. Abuse Treat. 66, 1-8.

Hayes, A.F., 2009. Beyond Baron and Kenny: Statistical mediation analysis in the new millennium. Commun. Monogr. 76, 408-420.

Hayes, A.F., 2013. Introduction to Mediation, Moderation, and Conditional Process Analysis: A Regression-Based Approach. The Guilford Press, New York.

Hayes, A.F., Rockwood, N.J., 2016. Regression-based statistical mediation and moderation analysis in clinical research: Observations, recommendations, and implementation. Behav. Res. Ther.

Hesse, M., Moran, P., 2010. Screening for personality disorder with the Standardised Assessment of Personality: Abbreviated Scale (SAPAS): Further evidence of concurrent validity. BMC Psychiatry 10, 10.

Hesse, M., Rasmussen, J., Pedersen, M.K., 2008. Standardised assessment of personality - a study of validity and reliability in substance abusers. BMC Psychiatry 8,7 .

Hester, R., Lubman, D.I., Yücel, M., 2010. The role of executive control in human drug addiction. Curr. Top. Behav. Neurosci. 3, 301-318.

Hofmann, W., Schmeichel, B.J., Baddeley, A.D., 2012. Executive functions and selfregulation. Trends Cogn. Sci. 16, 174-180.

Hopwood, C.J., Schade, N., Matusiewicz, A., Daughters, S.B., Lejuez, C.W., 2015. Emotion regulation promotes persistence in a residential substance abuse treatment. Subst. Use Misuse 50, 251-256.

Hudson, A., Jacques, S., 2014. Put on a happy face! Inhibitory control and socioemotional knowledge predict emotion regulation in 5- to 7-year-olds. J. Exp. Child Psychol. 123, 36-52.

IBM Corp, 2012. IBM SPSS Statistics for Windows, version 21.0. IBM Corp,, Armonk, NY.

Isquith, P.K., Roth, R.M., Gioia, G., 2013. Contribution of rating scales to the assessment of executive functions. Appl. Neuropsychol. Child 2, 125-132.

Jurado, M.B., Rosselli, M., 2007. The elusive nature of executive functions: A review of our current understanding. Neuropsychol. Rev. 17, 213-233.

Joormann, J., Stanton, C.H., 2016. Examining emotion regulation in depression: A review and future directions. Behav. Res. Ther. 86, 35-49. 
Karukivi, M., Vahlberg, T., Horjamo, K., Nevalainen, M., Korkeila, J., 2017. Clinical importance of personality difficulties: Diagnostically sub-threshold personality disorders. BMC Psychiatry 17, 16.

Kluwe-Schiavon, B., Sanvicente-Vieira, B., Kristensen, C.H., Grassi-Oliveira, R., 2013. Executive functions rehabilitation for schizophrenia: A critical systematic review. J. Psychiatr. Res. 47, 91-104.

Lubman, D.I., Yücel, M., Hall, W.D., 2007. Substance use and the adolescent brain: A toxic combination? J. Psychopharmacol. 21, 792-794.

Manning, V., Verdejo-García, A., Lubman, D.I., 2017. Neurocognitive impairment in addiction and opportunities for intervention. Curr. Opin. Behav. Sci. 13, 40-45.

Marceau, E.M., Berry, J., Lunn, J., Kelly, P.J., Solowij, N., 2017. Cognitive remediation improves executive functions, self-regulation and quality of life in residents of a substance use disorder therapeutic community. Drug Alcohol Depen. $178,150-158$.

Marceau, E.M., Lunn, J., Berry, J., Kelly, P.J., Solowij, N., 2016. The Montreal Cognitive Assessment (MoCA) is sensitive to head injury and cognitive impairment in a residential alcohol and other drug therapeutic community. J. Subst. Abuse Treat. 66, 30-36.

McClure, G., Hawes, D.J., Dadds, M.R., 2016. Borderline personality disorder and neuropsychological measures of executive function: A systematic review. Personal. Ment. Health 10, 43-57.

McRae, K., Jacobs, S.E., Ray, R.D., John, O.P., Gross, J.J., 2012. Individual differences in reappraisal ability: Links to reappraisal frequency, well-being, and cognitive control. J. Res. Pers. 46, 2-7.

Miyake, A., Friedman, N.P., 2012. The nature and organization of individual differences in executive functions: Four general conclusions. Curr. Dir. Psychol. Sci. 21, 8-14.

Miyake, A., Friedman, N.P., Emerson, M.J., Witzki, A.H., Howerter, A., Wager, T.D., 2000. The unity and diversity of executive functions and their contributions to complex "frontal lobe" tasks: A latent variable analysis. Cognitive Psychol. 41, 49100.

Mo, C., Deane, F., 2016. Reductions in craving and negative affect predict 3-month post-discharge alcohol use following residential treatment. Int. J. Ment. Health Addict. $14,761-774$.

Moran, P., Leese, M., Lee, T., Walters, P., Thornicroft, G., Mann, A., 2003.

Standardised Assessment of Personality - Abbreviated Scale (SAPAS): Preliminary validation of a brief screen for personality disorder. Brit. J. Psychiat. 183, 228-232.

Okita, K., Ghahremani, D.G., Payer, D.E., Robertson, C.L., Dean, A.C., Mandelkern, M.A., London, E.D., 2016. Emotion dysregulation and amygdala dopamine D2-type receptor availability in methamphetamine users. Drug Alcohol Depen. 161, 163-170. 
Osborne, T.L., Michonski, J., Sayrs, J., Welch, S.S., Anderson, L.K., 2017. Factor structure of the Difficulties in Emotion Regulation Scale (DERS) in adult outpatients receiving dialectical behavior therapy (DBT). J. Psychopathol. Behav. Assess. 39, 355-371.

Paschke, L.M., Dorfel, D., Steimke, R., Trempler, I., Magrabi, A., Ludwig, V.U., Schubert, T., Stelzel, C., Walter, H., 2016. Individual differences in self-reported selfcontrol predict successful emotion regulation. Soc. Cogn. Affect. Neurosci. 11, 11931204.

Pe, M.L., Koval, P., Houben, M., Erbas, Y., Champagne, D., Kuppens, P., 2015. Updating in working memory predicts greater emotion reactivity to and facilitated recovery from negative emotion-eliciting stimuli. Front. Psychol. 6, Article 372.

Polak, A.R., Witteveen, A.B., Reitsma, J.B., Olff, M., 2012. The role of executive function in posttraumatic stress disorder: A systematic review. J. Affect. Disord. 141, $11-21$

Roth, R.M., Isquith, P.K., Gioia, G., 2005. Behavioral Rating Inventory of Executive Function - Adult Version. Psychological Assessment Resources Inc., Lutz; Florida.

Schmeichel, B.J., Demaree, H.A., 2010. Working memory capacity and spontaneous emotion regulation: High capacity predicts self-enhancement in response to negative feedback. Emotion 10, 739-744.

Schmeichel, B.J., Tang, D., 2015. Individual differences in executive functioning and their relationship to emotional processes and responses. Curr. Dir. Psychol. Sci. 24, 93-98.

Schmeichel, B.J., Volokhov, R.N., Dernaree, H.A., 2008. Working memory capacity and the self-regulation of emotional expression and experience. J. Pers. Soc. Psychol. $95,1526-1540$.

Seligowski, A.V., Lee, D.J., Bardeen, J.R., Orcutt, H.K., 2015. Emotion regulation and posttraumatic stress symptoms: A meta-analysis. Cogn. Behav. Ther. 44, 87-102.

Sheehan, D.V., Lecrubier, Y., Sheehan, K.H., Amorim, P., Janavs, J., Weiller, E., Hergueta, T., Baker, R., Dunbar, G.C., 1998. The Mini-International Neuropsychiatric Interview (M.I.N.I): The development and validation of a structured diagnostic psychiatric interview for DSM-IV and ICD-10. J. Clin. Psychiatry 59, 22-33.

Smith, G.T., Cyders, M.A., 2016. Integrating affect and impulsivity: The role of positive and negative urgency in substance use risk. Drug Alcohol Depen. 163, Supplement 1, S3-S12.

Snyder, H.R., 2013. Major depressive disorder is associated with broad impairments on neuropsychological measures of executive function: A meta-analysis and review. Psychol. Bull. 139, 81-132.

Tamir, M., 2016. Why do people regulate their emotions? A taxonomy of motives in emotion regulation. Pers. Soc. Psychol. Rev. 20, 199-222. 
Tang, D., Schmeichel, B.J., 2014. Stopping anger and anxiety: Evidence that inhibitory ability predicts negative emotional responding. Cogn. Emot. 28, 132-142.

Toplak, M.E., West, R.F., Stanovich, K.E., 2013. Practitioner review: Do performance-based measures and ratings of executive function assess the same construct? J. Child Psychol. Psychiatry 54, 131-143.

Tull, M.T., Bardeen, J.R., DiLillo, D., Messman-Moore, T., Gratz, K.L., 2015. A prospective investigation of emotion dysregulation as a moderator of the relation between posttraumatic stress symptoms and substance use severity. J. Anxiety Disord. 29, 52-60.

Tull, M.T., Weiss, N.H., Adams, C.E., Gratz, K.L., 2012. The contribution of emotion regulation difficulties to risky sexual behavior within a sample of patients in residential substance abuse treatment. Addict. Behav. 10, 1084-1092.

Unoka, Z., Richman, M.J., 2016. Neuropsychological deficits in BPD patients and the moderator effects of co-occurring mental disorders: A meta-analysis. Clin. Psychol. Rev. 44, 1-12.

van Zutphen, L., Siep, N., Jacob, G.A., Goebel, R., Arntz, A., 2015. Emotional sensitivity, emotion regulation and impulsivity in borderline personality disorder: A critical review of fMRI studies. Neurosci. Biobehav. Rev. 51, 64-76.

Verdejo-García, A., 2016. Cognitive training for substance use disorders: Neuroscientific mechanisms. Neurosci. Biobehav. Rev. 68, 270-281.

von Hippel, W., Gonsalkorale, K., 2005. 'That is bloody revolting!': Inhibitory control of thoughts better left unsaid. Psychol. Sci. 16, 497-500.

Wechsler, D., 2008. Wechsler Adult Intelligence Scale. Pearson, Sydney; Australia.

Wen, Z., Fan, X., 2015. Monotonicity of effect sizes: Questioning kappa-squared as mediation effect size measure. Psychol. Methods 20, 193-203.

Wilcox, C.E., Adinoff, B., 2015. Using neuroimaging to improve emotion regulation treatments for substance use disorders. In: Feldstein Ewing, S.W., Witkiewitz, K., Filbey, F.M. (Eds.), Neuroimaging and Psychosocial Addiction Treatment. Palgrave Macmillan, London, UK.

Wilcox, C.E., Pommy, J.M., Adinoff, B., 2016. Neural circuitry of impaired emotion regulation in substance use disorders. Am. J. Psychiatry 173, 344-361.

Wills, T.A., Simons, J.S., Sussman, S., Knight, R., 2016. Emotional self-control and dysregulation: A dual-process analysis of pathways to externalizing/internalizing symptomatology and positive well-being in younger adolescents. Drug Alcohol Depen. 163, Supplement 1, S37-S45.

Witkiewitz, K., Wu, J., 2010. Emotions and relapse in substance use: Evidence for a complex interaction among psychological, social, and biological processes. In: Kassel, J.D. (Ed.), Substance Abuse and Emotion. American Psychological Association, Washington; DC. pp. 171-187. 
Zilverstand, A., Parvaz, M.A., Moeller, S.J., Goldstein, R.Z., 2016. Cognitive interventions for addiction medicine: Understanding the underlying neurobiological mechanisms. In: Hamed, E., Martin, P.P. (Eds.), Progress in Brain Research. Elsevier. pp. 285-304. 


\section{Figure Captions}

Figure 1. Models of EFs measures as predictors of Difficulties in Emotion Regulation Scale (DERS) scores, mediated by Standardised Assessment of Personality - Abbreviated Scale (SAPAS) scores. Confidence intervals (CIs) for the indirect effects are bias-corrected and accelerated (BCa) bootstrapped CIs based on 5000 samples.

Figure 1a. Model of task-switching performance as a predictor of DERS scores, mediated by SAPAS scores $(N=48)$.

Figure $1 b$. Model of WMI performance as a predictor of DERS scores, mediated by SAPAS scores $(N$ $=49$ ).

Figure 1c. Model of inhibition performance as a predictor of DERS scores, mediated by SAPAS scores $(N=48)$

Figure 1d. Model of Global Executive Composite (GEC) scores as a predictor of DERS scores, mediated by SAPAS scores $(N=49)$. 